\title{
COMPARATIVE EVALUATION OF METAL DAMAGE ON THE FREE LATERAL SUR- FACE OF SINGLE-LAYER AND THREE-LAYER STRIPS UNDER ROLLING
}

\author{
S.V. Smirnov, I.A. Veretennikova* \\ Institute of Engineering Science, Ural Branch of the Russian Academy of Sciences, 34 Komsomolskaya st., \\ Ekaterinburg, Russian Federation \\ *Corresponding author. E-mail: irincha@imach.uran.ru; address for correspondence: 34. Komsomolskaya st, 620049, \\ Ekaterinburg, Russian Federation; Tel.: +7 (343) 375-35-96; fax: +7 (343) 374-53-30
}

The paper compares the stress-strain state and damage accumulation in a steel constituting a "steel 20" single-layer strip and "12Cr18Ni10 Ti - steel $20-12 \mathrm{Cr} 18 \mathrm{Ni10Ti"} \mathrm{and} \mathrm{"copper} \mathrm{M1} \mathrm{-}$ steel 20 - copper M1" three-layer strips under rolling. The areas in the middle of the free lateral surface that are the most dangerous in terms of cohesive destruction are discussed. The finite el-ement method is used to simulate the rolling process. Kolmogorov's model of damage and the relation of peak plasticity to the stress state coefficient and the Lode coefficient are used for cal-culations. The analysis of the data shows a relationship between the configuration of the defor-mation zone and metal deformability. In particular, it is better to roll strips of steel 20 in large-diameter rolls and "copper M1 - steel 20 - copper M1" strips in small-diameter rolls. When "12Cr18Ni10Ti - steel 20 - 12Cr18Ni10Ti" strips ate rolled, the diameter of rolls has no essential effect on damage accumulation in the central layer. The proposed conclusions can be used only for the materials under study, since the mechanisms of damage accumulation for specific materials may vary depending on the peak plasticity diagram.

Keywords: multilayer metal, rolling, stress-strain state, damage.

DOI: $10.17804 / 2410-9908.2015 .4 .006-017$

\section{References}

1. Kobelev A.G., Lysak V.I., Chernyshev V.N. Proizvodstvo sloistykh kompozitsionnykh materialov [Production of Layered Composite Materials]. Moscow, Intermet Inzhiniring Publ., 2002, 496 p. (In Russian).

2. Trykov Ju.P., Shmorgun V.G., Gurevich L.M. Deformatsiya sloistykh kompozitov: Monografiya [Deformation of Layered Composites: Monograph]. Volgograd, VolGTU Publ., 2001, 242 p. (In Russian).

3. Smirnov S.V., Veretennikova I.A., Kamantsev I.S., Trushina E.B. Studying the destruction of the interface in an explosion-welded «Steel 08X18H10T + Steel 10» bimetal strip under plastic deformation. Proizvodstvo prokata, 2014, no. 7, pp. 14-19. (In Russian).

4. Smirnov, S.V. Veretennikova, I.A. Vichuzhanin, D.I. Modeling of delamination in multilayer metals produced by explosive welding under plastic deformation. Computational Continuum Mechanics, 2014, vol. 7, no. 4, pp. 398-411. http://dx.doi.org/10.7242/1999-6691/2014.7.4.38. (In Russian).

5. Kolmogorov V.L. Napryazheniya, deformatsii, razrushenie [Stresses, Strains, Fracture]. Moscow, Metallurgiya Publ., 1970, 229 p. (In Russian).

6. Bogatov A.A., Mizhiritsky O.I., Smirnov S.V. Resurs plastichnosti metallov pri obrabotke davleniem [Metal Plasticity Margin under Plastic Deformation]. Moscow, Metallurgiya Publ., 1984, 144 p. (In Russian).

7. Smirnov S.V., Golubkova I.A. Simulation of cold rolling by the finite element method. Zagotovitelnye proizvodstva $v$ mashinostroenii, 2010, no. 5, pp. 27-30. (In Russian).

8. Grudev A.P. Teoriya prokatki [Theory of Rolling]. Moscow, Intermet Inzhiniring Publ., 2001, 280 p. (In Russian). 
open-aCCess journal

9. Sorokin V.G., Volosnikova A.V., Vyatkin S.A. et al. Marochnik staley i splavov, pod obshch. red. V.G. Sorokina [V.G. Sorokin, ed. Book of Steel and Alloy Grades]. Moscow, Mashinostroenie Publ., 1989, 640 p. (In Russian).

10. Smirnov S., Domilovskaya T. Damage accumulation upon plastic deformation under monotonic loading. Russian metallurgy (Metally), 2002, no. 5, pp. 470-476.

11. Smirnov S., Domilovskaya T. Definition of the kinetic equation form for damage under the plastic deformation. Fatigue and Fracture of Engineering Materials and Structures, 2003, vol. 26 (4), pp. 373-379

12. Smirnov S.V., Shveikin V.P. Plastichnost i deformiruemost uglerodistoy stali [Plasticity and Deformability of Carbon Steel]. Ekaterinburg, UrO RAN Publ., 2008, 256 p. (In Russian).

13. Vichuzhanin D.I. Khotinov V.A. Smirnov S.V. The Effect of the Stress State on the Ultimate Plasticity of Steel X80. Diagnostics, Resource and Mechanics of materials and structures, 2015, iss. 1, pp. 73-89. URL: http://dream-journal.org/issues/2015-1/2015-1_21.html (accessed: 23.08.2015). http://dx.doi.org/10.17804/2410-9908.2015.1.073-089. (In Russian).

14. Smirnova S.V., Gladkovsky S.V. Mechanical properties and peculiarities of the fracture of layered metal composites after explosion welding and pack rolling. Aktualnye problemy sovremennoy nauki: Trudy 5-go Mezhdunarodnogo foruma. Yestestvennye nauki. Chasti 1-3: Matematika. Matematicheskoe modelirovanie. Mekhanika, 2010, pp. 214-218. (In Russian). 
Подана в журнал: 24.08.2015

УДК 621.7.011:539.374

DOI: $10.17804 / 2410-9908.2015 .4 .006-017$

\title{
СРАВНИТЕЛЬНАЯ ОЦЕНКА ПОВРЕЖДЕННОСТИ МЕТАЛЛА НА СВОБОДНОЙ БОКОВОЙ ПОВЕРХНОСТИ ПРИ ПРОКАТКЕ ОДНОСЛОЙНЫХ И ТРЕХСЛОЙНЫХ ПОЛОС
}

\author{
С.В.Смирнов, И.А. Веретенникова ${ }^{*}$
}

\begin{abstract}
Федеральное государственное бюджетное учреждение науки Институт машиноведения Уральского отделения Российской академии наук, ул. Комсомольская, 34, Екатеринбург, Российская Федерация

*Ответственный автор. Электронная почта: irincha@imach.uran.ru; Адрес для переписки: ул. Комсомольская, 34, 620049, Екатеринбург, Российская Федерация. Телефон: +7 (343) 375-35-96; Факс: 374-53-30
\end{abstract}

Проведено сравнение напряженно-деформированного состояния и накопления поврежденности стали 20 в составе однослойной полосы и трехслойных полос «12Х18Н10Т-сталь 20-12Х18Н10Т», «медь М1-сталь 20-медь М1» при прокатке. Рассмотрены наиболее опасные для когезионного разрушения участки на середине высоты свободной боковой поверхности. Моделирование осуществлено методом конечных элементов. Для расчетов использованы модель поврежденности В.Л. Колмогорова и зависимость предельной пластичности от показателя напряженного состояния и показателя Лоде. Установлено, что форма очага деформации $\ell / H_{c p}$ оказывает влияние на деформируемость металла. Расчеты показывают, что полосы из стали 20 целесообразно катать в валках большого диаметра, полосы «медь М1сталь 20-медь M1»- в валках меньшего диаметра. Диаметр валков при прокатке полосы «12Х18Н10Т-сталь 20-12X18Н10Т» не оказывает существенного влияния на накопление поврежденности в центральном слое. Данные выводы имеют частный характер, и закономерности накопления поврежденности для конкретных материалов могут изменяться в зависимости от диаграммы предельной пластичности.

Ключевые слова: слочстый листовой композиционный металл, прокатка, напряженно-деформированное состояние, поврежденность.

\section{1. Введение}

Одними из наиболее распространенных металлических композиционных материалов являются слоистые листовые материалы, состоящие из нескольких однородных или разнородных слоев. Важнейшее их преимущество в том, что для конкретных условий эксплуатации могут быть разработаны композиции с оптимальным комплексом служебных характеристик [1].

Основным процессом получения слоистых металлических материалов является сварка находящихся в контакте поверхностей металлов, в результате которой возникает прочное сцепление слоев. В дальнейшем полученные сваркой материалы часто подвергаются пластической деформации [2]. При этом для любого процесса пластического деформирования необходимо осуществлять прогнозирование поведения материала при обработке и в процессе дальнейшей эксплуатации. Как показывают исследования, закономерности деформировании монолитных материалов отличаются от процесса деформирования многослойных листовых материалов [3]. Разрушение таких материалов может происходить за счет расслоения сварных границ и по механизму когезионного разрушения, т. е. за счет исчерпания ресурса пластичности металла слоев, составляющих композит. Вопросы моделирования и прогнозирования расслоения на границе соединения разнородных материалов были рассмотрены нами в работе [4]. Статья посвящена сравнению особенностей напряженнодеформированного состояния (НДС) и накопления поврежденности для наиболее опасных

Smirnov S. V. et al. / Comparative evaluation of metal damage on the free lateral surface of single-layer and 
для разрушения участков металла, расположенных на середине высоты свободной боковой поверхности трехслойных полос по сравнению с монолитными.

Модели механики поврежденности преимущественно используются для описания процессов разрушения монолитного материала. Вопрос применения подобных моделей для оценки поврежденности и разрушения составляющих металлических композиционных материалов специально не изучался. Наиболее последовательно в настоящее время изучена применимость для прогнозирования разрушения металлов при пластической деформации моделей, разработанных научной школой В.Л. Колмогорова $[5,6]$, линейный вариант которых был использован в данной статье. В качестве объекта исследования выбраны образцы, полученные сваркой взрывом. Пластическая деформация осуществлялась прокаткой в гладких валках. Рассматривалось поведение материала на боковой границе прокатываемой полосы. Выбор данных слоистых материалов обусловлен их широким применением в промышленности и удобством проведении модельных экспериментов. В качестве метода моделирования был выбран метод конечных элементов, являющийся одним из наиболее распространенных в настоящее время методов численного решения трехмерных нелинейных задач механики деформируемого твердого тела.

\section{2. Постановка задачи и исследуемые материалы}

Моделирование прокатки осуществляли в программе LS-DYNA, которая предназначена для решения высоко-нелинейных технологических задач механики. Возможность адекватного моделирования прокатки полосы средствами программы LS-DYNA была описана в статье [7], где сравнивались результаты исследований контактных напряжений при холодной прокатке алюминиевых образцов разной высоты на лабораторном стане при установившейся стадии процесса, полученные численным и натурным экспериментами, показана применимость конечно-элементной модели для целей оценки напряженно-деформируемого состояния и прогнозирования поврежденности и разрушения при прокатке.

Твердотельные модели полосы и валков создавали средствами программы ANSYS/LS-DYNA. При построении конечно-элементной модели прокатки принимали, что в первоначальный момент времени захват уже произошел, т. е. полоса уже находится в валках.

Упорядоченную сетку строили путем деления геометрических элементов модели на некоторое число частей с использованием встроенного алгоритма разумного выбора размеров конечных элементов, с учетом кривизны поверхности и наилучшего отображения ее реальной геометрии, что позволило уменьшить число элементов неудовлетворительной формы и прерываний процедуры построения сетки. При разбиении сетки использовали объемные конечные 8-узельные элементы SOLID186. Упругой деформацией валков пренебрегали, принимая допущение, что они абсолютно жесткие. В связи с этим более мелкое разбиение конечно-элементной сетки было выполнено не во всем объеме валков, а только на их поверхностях, непосредственно контактирующих с заготовкой.

Контакт полосы с валками задавали в виде контактных пар «абсолютно жесткое тело - деформируемое тело». При этом полагали, что для контактных поверхностей справедлив закон Амонтона - Кулона со значением коэффициента трения 0,1. Скорость вращения валков составляла 0,3 м/с. Для материалов полосы использовали изотропную упругопластическую модель среды с изотропным упрочнением. Материалы в слоистой полосе соединялись без возможности проскальзывания и разъединения с помощью контактной опции BONDING. Для минимизации функционала вариационного принципа Лагранжа был выбран метод Бройдена-Флетчера-Гольдфарба-Шэнно, являющийся одной из модификаций метода Ньютона. Для решения системы линейных уравнений на очередной итерации поиска минимума функционала использовали метод сопряженных градиентов и неявную схему интегрирования по временной области. Для контроля устойчивости численного решения в ходе расчетов выполняли проверку энергетического баланса системы: контролировали работу внешних

Smirnov S. V. et al. / Comparative evaluation of metal damage on the free lateral surface of single-layer and three-layer strips under rolling. 
сил, энергию формоизменения, потенциальную энергию упругой деформации заготовки, кинетическую энергию заготовки, рассеяние энергии в контакте заготовки с валками.

Представленная конечно-элементная модель процесса прокатки достаточно универсальна, позволяет анализировать практически все необходимые параметры напряженнодеформированного состояния при широком варьировании условий технологического процесса. Задачи прокатки по различным схемам различаются лишь геометрической постановкой.

В теории холодной прокатки принято [8], что напряженно-деформированное состояние зависит от двух безразмерных параметров - относительного обжатия $\varepsilon=\frac{H_{0}-H_{1}}{H_{0}} 100 \%$ и фактора очага деформации $\ell / H_{c p}\left(\ell-\right.$ длина геометрического очага деформации; $H_{c p}=$ 0,5 $\left(H_{0}+H_{l}\right)$ - средняя высота полосы при прокатке; $H_{0}$ и $H_{l}$ начальная и текущая высота полосы соответственно). Величина $\ell$ рассчитывается по формуле $[8] \ell=\sqrt{0,5 D_{B}\left(H_{0}-H_{1}\right)}$, где $D_{\text {в }}$ - диаметр валка.

При моделировании принимали, что относительное обжатие при прокатке $\varepsilon=10 \%$ во всех рассмотренных вариантах прокатки. Изменение напряженно-деформированного состояния осуществлялось за счет варьирования фактора формы очага деформации $\ell / H_{c p}$. При моделировании варьирование $\ell / H_{c p}$ достигали за счет изменения диаметра валков $D_{\text {в }}=250 ; 130$; 50 мм. Значения $\ell / H_{c p}$, реализуемые в условиях вычислительного эксперимента, приведены в табл. 1.

Таблица 1 - Данные к моделированию прокатки

\begin{tabular}{|c|c|c|}
\hline$D_{\mathrm{B}}, \mathrm{MM}$ & $\ell, \mathrm{MM}$ & $\ell / H_{\mathrm{cp}}$ \\
\hline 250 & 10,60 & 1,20 \\
\hline 130 & 7,65 & 0,89 \\
\hline 50 & 4,74 & 0,55 \\
\hline
\end{tabular}

Процесс прокатки с $\ell / H_{c p}=0,55 \ldots . .1,2$ соответствует случаю прокатки полос средней толщины, когда средние по высоте слои металла деформируются наиболее интенсивно, в приконтактных слоях образуются зоны затрудненной деформации, боковые кромки приобретают выпуклую форму [8].

Рассматривали сталь 20 в составе однослойной и трехслойных полос со следующим сочетанием слоев «12Х18Н10Т-сталь 20-12X18Н10Т», «медь М1-сталь 20-медь М1». Выбор материалов трехслойных полос обусловлен разными значениями предела текучести и склонностями к деформационному упрочнению стали 12Х18Н10Т и меди М1. Во всех случаях исходная толщина полосы была одинаковой и составляла $H_{0}=9$ мм, первоначальная ширина всех полос $-B_{0}=30$ мм. Данные по слоям приведены в табл. 2.

Сопротивление деформации $\sigma_{s}(\varepsilon)$ в условии пластичности Мизеса задавали в табличной форме в соответствии с данными, полученными после растяжения на универсальной испытательной машине INSTRON8801 образцов, изготовленных из исходных полос сталей 20, 12Х18Н10Т и меди M1 (рис. 1). Упругие свойства взяли из справочных данных [9]:для стали $12 \mathrm{X} 18 \mathrm{H} 10 \mathrm{~T}$ значения нормального модуля упругости $E=210$ ГПа и коэффициента Пуассона $v=0,3$; для стали $20-E=200$ ГПа, $v=0,28$; для меди М1 $-E=125$ ГПа, $v=0,34$.

Таблица 2 - Соотношение слоев в многослойных материалах

\begin{tabular}{|c|c|}
\hline Соотношение слоев & Толщина слоев (в скобках), мм \\
\hline 12Х18Н10Т-сталь20-12Х18Н10Т & $9(2+5+2)$ \\
\hline медь М1-сталь 20-медь М1 & $9(2+5+2)$ \\
\hline
\end{tabular}

Smirnov S. V. et al. / Comparative evaluation of metal damage on the free lateral surface of single-layer and three-layer strips under rolling. 


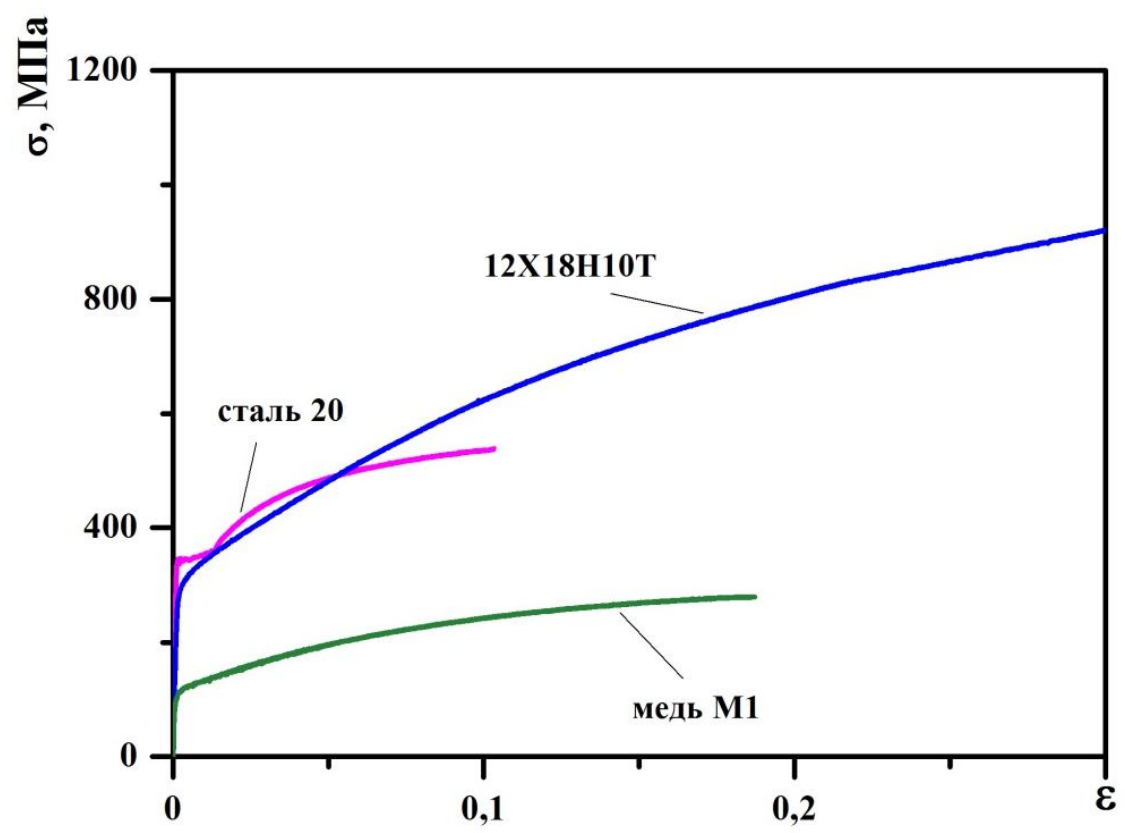

Рис.1. Кривые сопротивления деформации материалов в исходном состоянии

\section{3. Исследование напряженно-деформированного состояния составляющих многослой- ной полосы}

В феноменологической теории поврежденности в качестве интегральных характеристик напряженного состояния используют показатель напряженного состояния

$$
k=\sigma / T
$$

и показатель Лоде

$$
\mu_{\sigma}=2 \frac{\sigma_{22}-\sigma_{33}}{\sigma_{11}-\sigma_{33}}-1
$$

В формулы для расчета показателей входят следующие величины:

- $\sigma=\frac{1}{3}\left(\sigma_{11}+\sigma_{22}+\sigma_{33}\right)-$ среднее нормальное напряжение $\left(\sigma_{11}, \sigma_{22}, \sigma_{33}\right.$ - главные нормальные напряжения);

- интенсивность касательных напряжений $T=\sqrt{0,5 s_{i j} s_{i j}}\left(s_{i j}-\right.$ компоненты девиатора напряжений).

В качестве обобщенной характеристики накопленной деформации материальной частицы использовали степень деформации сдвига $\Lambda$, рассчитываемой вдоль траектории движения частицы [5]. Приращение $d \Lambda$ рассчитывается как $d \Lambda=H d \tau$, где $\tau$ - время деформирования, $H=\sqrt{2 \xi_{i j} \xi_{i j}}-$ интенсивность скоростей деформации сдвига, где $\xi_{i j}-$ компоненты тензора скорости деформации.

Вид конечно-элементной модели прокатки трехслойной полосы представлен на рис. 2. Проведенное моделирование позволило определить формоизменение полос в очаге деформации и во внеконтактных зонах, рассчитать компоненты тензоров напряжений и деформаций, накопленную степень деформации сдвига, определить параметры $k$ и $\mu_{\sigma}$, от которых зависит накопление поврежденности материала листов при когезионном разрушении. 
open-recess journal

Характеристики напряженно-деформированного состояния рассчитывали для материальных точек, траектория движения которых соотносится со средней линией свободной боковой поверхности полосы (слоя). Сравнивали напряженно-деформированное состояние стали 20 в трехслойной и однослойной полосах в одном и том же месте. Для этого выбирали узел сетки, расположенный на расстоянии, равном одной длине геометрического очага деформации $\ell$ от начала полосы, и фиксировали изменения напряжений и деформаций при прохождении узла через геометрический очаг деформации (рис.3).

Результаты расчетов представлены на рис. 4, где данные относятся к геометрическому очагу деформации, поэтому при $x / \ell=0$ металл уже претерпевает некоторую деформацию во внеконтактных зонах [8].
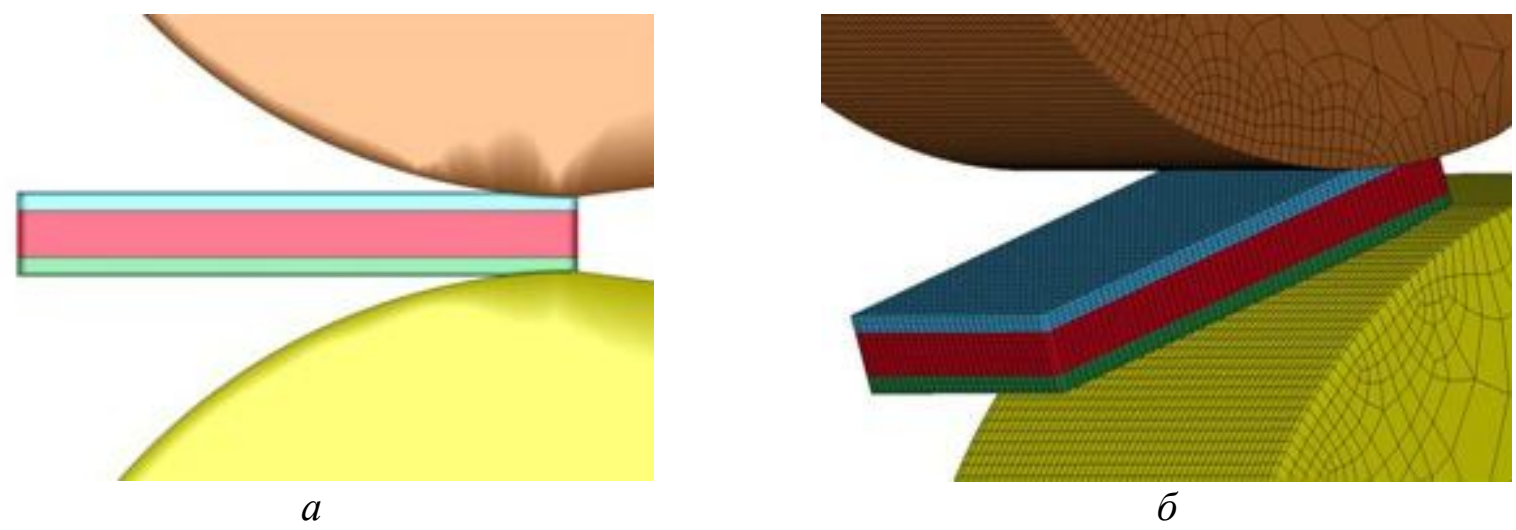

6

Рис. 2. Вид конечно-элементной модели при прокатке трехслойной полосы

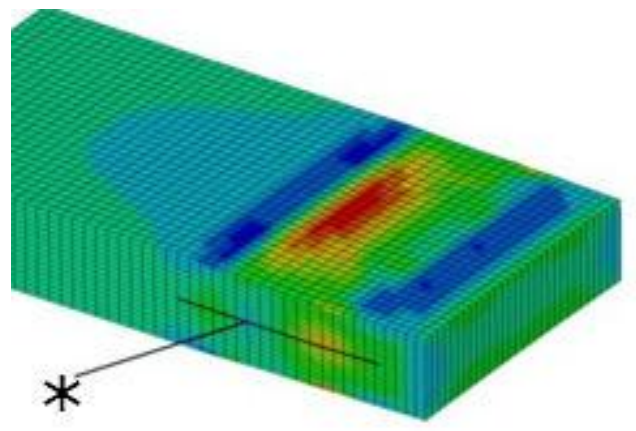

$a$

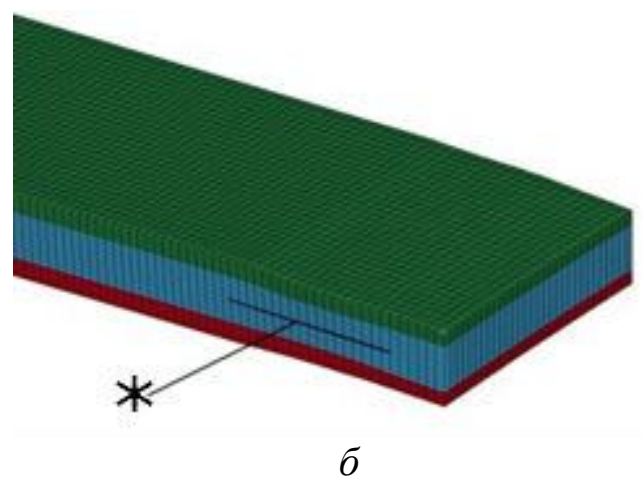

$\sigma$

Рис.3. Траектории материальных частиц *, для которых производили расчет параметров напряженно-деформированного состояния:

$a$ - однослойная полоса; $\sigma$ - трехслойная полоса

Напомним также, что при $k<0$ частица металла испытывает состояние сжатия, при $k>0$ - растяжения, а при $k=0$ реализуется схема плоского напряженного состояния.

Основным концептуальным и расчетным параметром в феноменологической теории разрушения [5] является поврежденность $\omega$. До деформации $\omega=0$ в момент возникновения макротрещины $\omega=1$, а промежуточные значения $\omega$ характеризуют уровень накопления в металле деформационных микродефектов сплошности. Установлено, что существуют некоторые пороговые значения поврежденности, свыше которой в деформируемом металле образуются незалечиваемые рекристаллизационным отжигом микродефекты $\left(\omega=\omega^{*}=0,2-0,4\right)$ и микротрещины, видимые невооруженным глазом $\left(\omega=\omega^{* *}=0,6-0,8\right)[6]$. Деформация на боковой внеконтактной поверхности на продольной оси симметрии не носит знакопеременный характер, следовательно, поврежденность может быть рассчитана с использованием линейной модели [5] по формуле: 

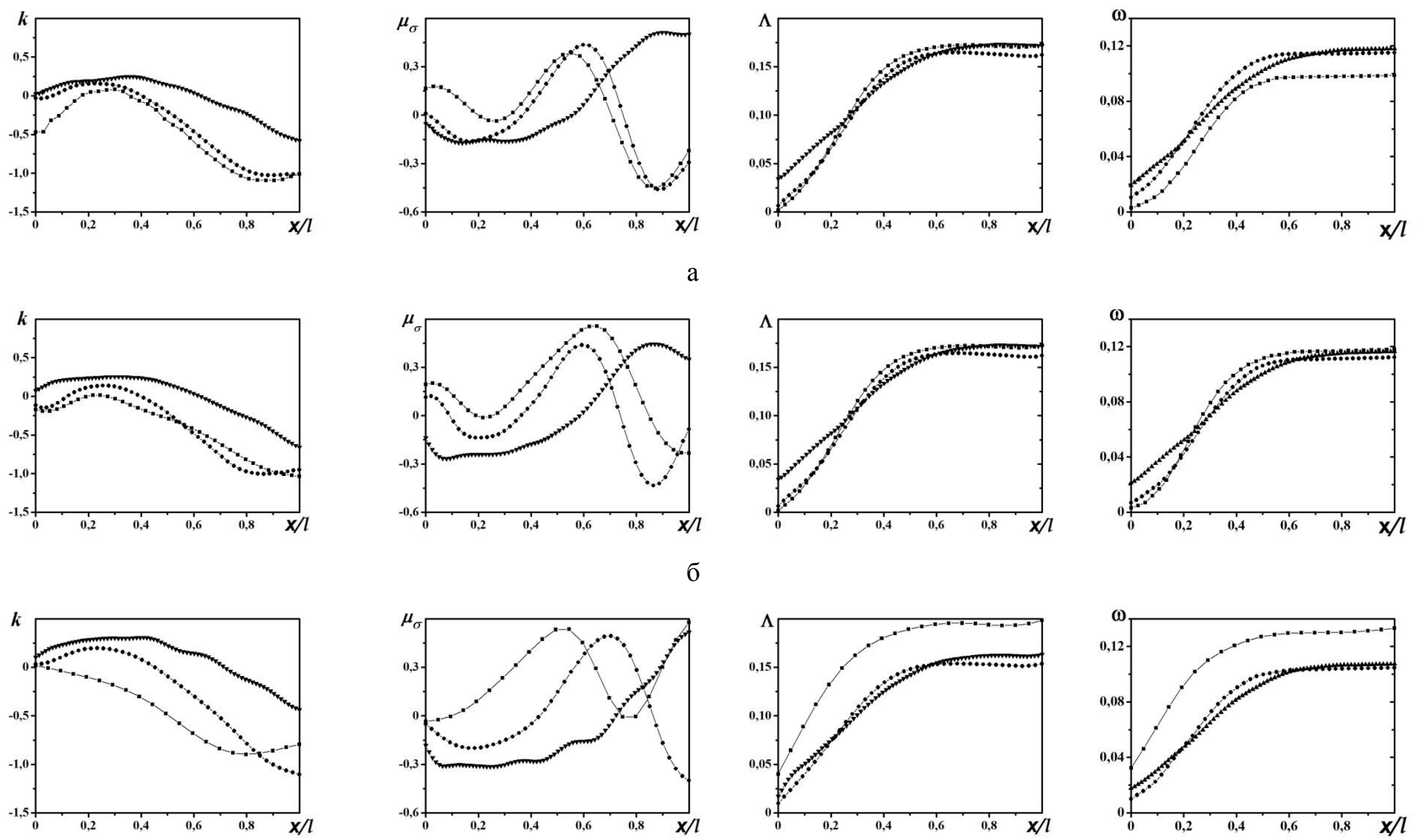

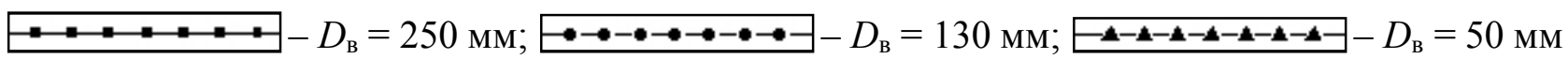

Рис. 4. Изменения показателей для стали 20 вдоль геометрического очага деформации: $a-$ на боковой поверхности однослойной полосы; б - на боковой поверхности полосы в материале «12X18Н10Т-сталь 20-12X18Н10Т»; в - на боковой поверхности полосы в материале «медь М1-сталь 20-медь М1»

Smirnov S. V. et al. / Comparative evaluation of metal damage on the free lateral surface of single-layer and three-layer strips under rolling. 


$$
\omega=\int_{0}^{\Lambda} \frac{d \Lambda}{\Lambda_{p}\left(k, \mu_{\sigma}\right)}
$$

где $\Lambda_{p}\left(k, \mu_{\sigma}\right)$ - пластичность металла [6].

Адекватность линейной модели при расчете поврежденности в условиях простого монотонного нагружения была экспериментально доказана по результатам исследования изменения плотности металлов при деформировании $[10,11]$.

Изменение показателей $k, \mu_{\sigma}$ и степени деформации сдвига $\Lambda$ рассчитывается по формулам (1), (2) вдоль траектории движения рассматриваемой частицы металла. Функция $\Lambda_{p}(k$, $\mu_{\sigma}$ ) определяется экспериментально при проведении серии механических испытаний, в которых показатели $k, \mu_{\sigma}$ принимают некоторые разные значения, образцы подвергаются деформированию до разрушения. Геометрическая интерпретация данной функции имеет название диаграммы предельной пластичности. Для расчетов поврежденности, проведенных в данной статье, была использована диаграмма пластичности (рис. 5), построенная по результатам экспериментов, проведенных А.В. Тропотовым по растяжению и кручению цилиндрических образцов в камере с регулируемым давлением жидкости, осадке цилиндрических образцов и растяжению цилиндрических образцов с кольцевыми концентраторами напряжений [6].

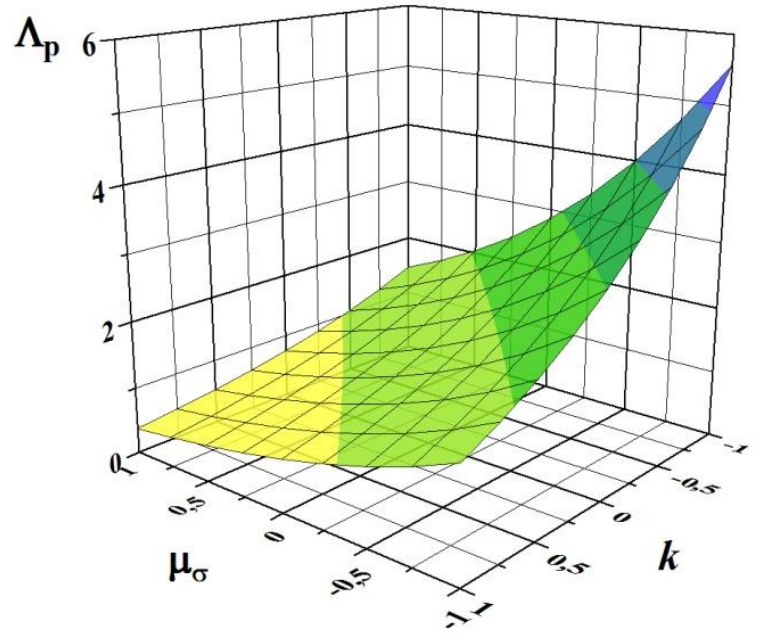

Рис. 5. Диаграмма предельной пластичности для стали 20

Аналитически диаграмма предельной пластичности была аппроксимирована функцией

$$
\Lambda_{p}\left(k, \mu_{\sigma}\right)=\exp \left[b_{1}+b_{2} \mu_{\sigma}+\left(b_{3}+b_{4} \mu_{\sigma}\right) k\right]
$$

где $b_{1}=0,401, b_{2}=-0,649, b_{3}=-0,699, b_{4}=-0,030-$ эмпирические коэффициенты.

Из рис. 5 следует, что предельная пластичность уменьшается с увеличением показателей $k$ и $\mu_{\sigma}$. Наибольшую предельную пластичность при фиксированном значении $k$ сталь 20 имеет в условиях осесимметричного растяжения $\left(\mu_{\sigma}=-1\right)$, что характерно для углеродистых сталей [122].

Из рис. 4 следует, что практически все накопление поврежденности происходит в первой половине очага деформации при $x / l<0,6$, которой соответствует положение зоны отставания, известной в теории продольной прокатки, в которой происходит основное обжатие и накопление степени деформации сдвига. Поэтому, в соответствии с формулой (3), изменение характеристик НДС в зоне отставания определяет закономерности накопления поврежденности и исчерпание ресурса пластичности по механизму когезионного разрушения ма- 
териала при прокатке. В табл. 3 приведены среднеинтегральные значения характеристик НДС в зоне отставания, рассчитанные для всех выполненных вариантов моделирования.

Таблица 3 - Средние значения характеристик НДС в зоне отставания

\begin{tabular}{|c|c|c|c|c|c|}
\hline Материал & $\begin{array}{c}\text { Диаметр } \\
\text { валков, мм }\end{array}$ & $k_{c p}$ & $\left(\mu_{\sigma}\right)_{c p}$ & $\Lambda$ & $\omega$ \\
\hline \multirow{2}{*}{ сталь 20 } & 250 & $-0,20$ & 0,18 & 0,14 & 0,10 \\
\cline { 2 - 6 } & 130 & $-0,02$ & 0,04 & 0,17 & 0,11 \\
\cline { 2 - 6 } & 50 & 0,14 & $-0,08$ & 0,17 & 0,12 \\
\hline \multirow{2}{*}{ 12X18Н10Т-сталь20-12X18H10T } & 250 & $-0,17$ & 0,21 & 0,17 & 0,12 \\
\cline { 2 - 6 } & 130 & $-0,07$ & 0,08 & 0,16 & 0,11 \\
\cline { 2 - 6 } & 50 & 0,17 & $-0,15$ & 0,17 & 0,12 \\
\hline \multirow{2}{*}{ медь М1-сталь 20-медь M1 } & 250 & $-0,27$ & 0,26 & 0,19 & 0,13 \\
\cline { 2 - 6 } & 130 & 0,04 & $-0,03$ & 0,15 & 0,10 \\
\cline { 2 - 6 } & 50 & 0,24 & $-0,27$ & 0,16 & 0,11 \\
\hline
\end{tabular}

Деформация слоев металла, расположенных ближе к контактной поверхности, больше чем у слоев, расположенных на середине толщины полосы. Это вызывает возникновение растягивающих продольных и поперечных напряжений и показатель $k$ имеет положительные значения. Значительное влияние на значения показателей $k$ и $\mu_{\sigma}$ имеет диаметр валков. При возрастании $\ell / H_{c p}$ с увеличением диаметра валков показатель $k$ приобретает отрицательные значения, что свидетельствует о преобладании сжимающих напряжений. В отличие от идеального случая плоской прокатки, когда отсутствуют уширение полосы и искривление профиля свободной боковой поверхности, там возникает схема трехосного напряженного состояния и показатель Лоде $\mu_{\sigma}$ отличен от 0.

Для полосы «медь М1-сталь 20-медь М1» увеличение степени накопленной деформации при прокатке в валках большего диаметра приводит к увеличению накопленной поврежденности срединных слоев по сравнению с прокаткой полос из стали 20 и композиции «12Х18Н10Т-сталь 20-12Х18Н10Т», а использование валков меньшего диаметра приводит к противоположному эффекту.

Анализ данных табл. 3 позволил определить некоторые закономерности накопления поврежденности стали 20 на свободной боковой поверхности однослойных и трехслойных полос. Видно, что наибольшая величина поврежденности накапливается при прокатке полосы «медь М1-сталь 20-медь М1» в валках большого диаметра. Этому способствует увеличение накопленной степени деформации сдвига и наибольшее, по сравнению с другими вариантами прокатки, среднее значение показателя Лоде $\mu_{\sigma}$. Причиной увеличения степени накопленной деформации являются большие значения степени обжатия и уширения менее прочных периферийных медных слоев, которые дополнительно «тянут» центральный стальной слой. Совместное отрицательное влияние на накопление поврежденности обоих отмеченных факторов превалирует над положительным влиянием увеличения сжимающих напряжений, характеризуемое уменьшением показателя $k$. Заметим, что если бы не учитывалось влияние на предельную пластичность схемы напряженного состояния, характеризуемое показателем $\mu_{\sigma}$, то сделанный выше вывод был бы противоположным.

Учитывая выявленное влияние фактора формы очага деформации $\ell / H_{c p}$, проявляющееся в данном случае через изменение диаметра валков, на деформируемость исследованных композиций, можно считать, что полосы из стали 20 целесообразно катать в валках большого диаметра, полосы «медь М1-сталь 20- медь М1»- в валках меньшего диаметра, а диаметр валков при прокатке полосы «сталь 12Х18Н10Т- сталь 20-сталь 12Х18Н10Т» не оказывает существенного влияния на накопление поврежденности в центральном слое из стали 20. Следует отметить, что влияние схемы напряженного состояния на предельную пластичность

Smirnov S. V. et al. / Comparative evaluation of metal damage on the free lateral surface of single-layer and three-layer strips under rolling. 
для разных материалов может существенно различаться, а зависимость $\Lambda_{p}\left(k, \mu_{\sigma}\right)$ иметь немонотонный характер. Ряд примеров диаграмм пластичности с такими особенностям приведен в работе [13]. Поэтому выводы, сделанные выше, имеют частный характер и закономерности накопления поврежденности для конкретных материалов могут изменяться в зависимости от того, какую они имеют диаграмму предельной пластичности.

Для оценки адекватности выполненного моделирования поврежденности были использованы результаты исследований многопроходной прокатки трехслойных полос «12Х18Н10Т-сталь 20-12X18Н10Т», изготовленныХ методом сварки взрывом [14]. Прокатка осуществлялась на стане 200 дуо-кварто ИМАШ УрО РАН в гладких валках диаметром 250 мм с разовыми обжатиями за проход $10 \%$. В результате исследований авторами было установлено, что разрушение центрального слоя происходит в процессе 8-го прохода прокатки при суммарном обжатии около $75 \%$. Как следует из табл. 3, за один проход с $10 \%$ обжатием величина поврежденности составляет $\omega=0,12$. Если считать, что в каждом проходе прокатки приращение поврежденности составляет такую же величину, то тогда за 8 проходов суммарная величина поврежденности будет $\omega=0,96$ при критериальном значении поврежденности в момент разрушения $\omega=1$. Это свидетельствует о хорошем соответствии результатов расчетов экспериментам и подтверждает адекватность прогнозирования когезионного разрушения при прокатке слоистых металлических материалов, используя концептуальный и математический аппарат механики поврежденности.

\section{4. Заключение}

1. Модели механики поврежденности позволяют осуществлять адекватное моделирование процессов поврежденности и исчерпания ресурса пластичности по механизму когезионного разрушения при прокатке полос из слоистых металлических материалов.

2. При выборе режимов прокатки слоистых полос исходя из критерия минимизации накопленной поврежденности следует учитывать влияние фактора формы очага деформации $\ell / H_{c p}$ на деформируемость металла. С учетом этого было установлено, что полосы из стали 20 целесообразно катать в валках большого диаметра, полосы «медь М1-сталь 20-медь М1» - в валках меньшего диаметра. Диаметр валков при прокатке полосы «12Х18Н10Т-сталь 20$12 \mathrm{X} 18 \mathrm{H} 10 \mathrm{~T} »$ не оказывает существенного влияния на накопление поврежденности в центральном слое из стали 20.

3. Так как влияние схемы напряженного состояния на предельную пластичность для разных материалов может существенно отличаться, а зависимость $\Lambda_{p}\left(k, \mu_{\sigma}\right)$ иметь немонотонный характер, то предыдущий вывод имеет частный характер, а закономерности накопления поврежденности для конкретных материалов могут изменяться в зависимости от того, какую они имеют диаграмму предельной пластичности.

\section{Благодарность}

Исследование выполнено при частичной финансовой поддержке гранта РФФИ и Правительства Свердловской области (проект № 13-08-96061).

\section{Литература}

1. Кобелев А. Г., Лысак В. И., Чернышев В. Н. Производство слоистых композиционных материалов. - М. : Интермет Инжиниринг, 2002. - 496 с.

2. Трыков Ю. П., Шморгун В. Г., Гуревич Л. М. Деформация слоистых композитов: монография. - Волгоград : ВолГТУ, 2001. - 242 с.

3. Исследование разрушения границы соединения слоев у полученной сваркой взрывом биметаллической полосы «08X18Н10Т-сталь 10» при прокатке / С. В. Смирнов, И. А. Веретенникова, И. С. Каманцев, Е. Б. Трушина // Производство проката. - 2014. - №7. C. 14-19.

Smirnov S. V. et al. / Comparative evaluation of metal damage on the free lateral surface of single-layer and three-layer strips under rolling. 
open-access journal

4. Смирнов С. В., Веретенникова И. А., Вичужанин Д. И. Моделирование расслоения при пластической деформации биметаллического материала, полученного сваркой взрывом // Вычислительная механика сплошных сред. - 2014. - № 4. - С. 398-411.

5. Колмогоров В. Л. Напряжения, деформации, разрушение. - М. : Металлургия, 1970. $229 \mathrm{c}$.

6. Богатов А. А., Мижирицкий О. И., Смирнов С.В. Ресурс пластичности металлов при обработке давлением. - М. : Металлургия, 1984. - 144 с.

7. Смирнов С. В., Голубкова И. А. Моделирование процесса холодной прокатки тонких полос методом конечных элементов // Заготовительные производства в машиностроении. 2010. - №5. - С. 27-30.

8. Грудев А. П. Теория прокатки. - Изд. 2-е перераб. и доп. - М. : Интермет Инжиниринг, 2001. -280 с.

9. Марочник сталей и сплавов / В. Г. Сорокин, А. В. Волосникова, С. А. Вяткин и др. / под общ. ред. В. Г. Сорокина. - М. : Машиностроение, 1989. - 640 с.

10. Smirnov S., Domilovskaya T. Damage accumulation upon plastic deformation under monotonic loading // Russian metallurgy (Metally). - 2002. - № 5. - P. 470-476.

11. Smirnov S., Domilovskaya T. Definition of the kinetic quationform for damage under the plastic deformation // Fatigue and Fracture of Engineering Materials and Structures. - 2003. V. 26. - P. 373-379.

12. Смирнов С. В., Швейкин В. П. Пластичность и деформируемость углеродистой стали. - Екатеринбург : УрО РАН, 2008. - 256 с.

13. Vichuzhanin D. I. Khotinov V. A. Smirnov S. V. The Effect of the Stress State on the Ultimate Plasticity of Steel X80 [Electronic resource] // Diagnostics, Resource and Mechanics of materials and structures. - 2015. - Iss. 1. - P. 73-89. - URL: http://dream-journal.org/issues/2015-1/20151_21.html (accessed: 23.08.2015). http://dx.doi.org/10.17804/2410-9908.2015.1.073-089.

14. Смирнова С. В., Гладковский С. В. Механические свойства и особенности разрушения слоистых металлических композитов после сварки взрывом и пакетной прокатки // Актуальные проблемы современной науки: Труды 5-ого Международного форума. Естественные науки. Части 1-3: Математика. Математическое моделирование. Механика. - Самара: СамГТУ. - 2010. - С. 214-218. 